\title{
Distinguishing mechanisms of morphological instabilities in phase change materials during switching
}

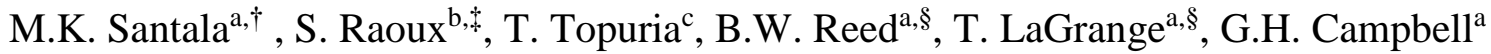 \\ ${ }^{1}$ Condensed Matter and Materials Division, Lawrence Livermore National Laboratory, \\ Livermore, CA 94551, USA \\ ${ }^{2}$ IBM T.J. Watson Research Center, Yorktown Heights, NY 10598, USA \\ ${ }^{3}$ IBM Research - Almaden, San Jose, CA 95120, USA
}

\section{Introduction}

The thermal stability of thin films and nanostructures is important for performance and reliability of materials for many applications, including memory based on phase change materials (PCMs). Chalcogenide-based PCMs, such as $\mathrm{Ge}_{2} \mathrm{Sb}_{2} \mathrm{Te}_{5}$ and GeTe, are important for use in optical recording media and non-volatile resistance-based memory devices [1], which exploit the distinct optical and electrical properties of the amorphous and crystalline phases. For memory applications, switching between the amorphous and crystalline phases is achieved within a few nanoseconds by laser or Joule heating [1]. During crystallization the amorphous PCM may be heated well above its glass transition temperature and during amorphization the melting temperature of the crystalline PCM is exceeded $[2,3]$. Heating induces an increase in atomic mobility which enables rapid phase transformations, but which can also induce morphological changes adversely affecting device performance and reliability. Some studies [46] have addressed device reliability by observing changes in microstructure and nanostructure morphology during heat-induced phase switching. Morphology changes leading to void

\footnotetext{
${ }^{\dagger}$ Corresponding author. E-mail address: santala1@1lnl.gov

$\ddagger$ Current address: Helmholtz-Zentrum Berlin für Materialien und Energie GmbH, Berlin, Germany

$\S$ Current address: Integrated Dynamic Electron Solutions, Pleasanton, CA 94588, USA
} 
formation have been observed in electrically-switched GeTe nanowires [5]. Morphological defects have been observed in in-line PCM cells due to faceting of grains during crystallization of the amorphous phase [6]. Atomic force microscopy experiments show that during a full switching cycle (crystal to amorphous to crystalline) the topography of a PCM thin film may not return to its original shape [4].

Although morphology changes can have a dramatic effect on the performance of PCM devices $[5,6]$, relatively few studies of the physical mechanisms underlying morphology changes and dewetting have been published about PCMs. This may be, in part, due to the short timescales (ns) under which these morphological changes occur in PCMs when rapidly heated by laser or Joule heating, making the relevant experimental measurements difficult to accomplish. The processes leading to hole formation in thin films of Te and Ge-Te alloys have been considered by Kivits et al. [7] in the context of intentional hole formation for optical data storage. They considered how gradients in surface tension and pressure induced by localized laser heating may lead to film thinning and rupture at the center of the laser-affected area and also considered the role of defects and pre-existing film defects in hole formation. The aforementioned mechanisms may play a role in the development of thickness variations and hole formation during laser amorphization and crystallization in thin films of $\mathrm{Ge}_{2} \mathrm{Sb}_{2} \mathrm{Te}_{5}$ shown in Figure 1. However, the patterns of the thickness variations in Figure $1 \mathrm{a}$ and $\mathrm{b}$ and the more complex patterns of laserinduced thickness changes observed with in situ transmission electron microscopy (TEM) during laser crystallization of amorphous GeTe thin films [8] suggest that other types of thin films instabilities may be operational. Here we report on initial results using nanosecond time-resolved TEM to investigate the physical mechanisms causing thin film instabilities in PCM thin films during laser annealing. 
Thin film instabilities and dewetting have been studied very extensively in other material systems [9-24], due to their importance in an enormous range of technologies (e.g. paints, coatings, electronics, dielectric layers, thermal barrier coatings, etc.). Instabilities and dewetting in liquid thin films can be initiated in several ways [9-11]. They may begin at pre-existing defects, such as pinholes, or may be nucleated [11] by a local variation in surface tension or film pressure which cause local thinning of the film. Instabilities can be induced by van der Waals interactions requiring no nucleation event $[9,12]$. It has been shown that in free-standing [9] or supported [12] very thin liquid films $(<100 \mathrm{~nm})$, thickness fluctuations above a critical wavelength, $\lambda_{c}$, will grow spontaneously due to the disjoining pressure arising from van der Waals interactions (below $\lambda_{c}$ the film is still effectively stabilized by surface tension). The wavelength of the thickness fluctuation with the fastest growing amplitude, $\lambda_{\mathrm{m}}=\sqrt{ } 2 \lambda_{\mathrm{c}}$, determines the characteristic length scale of the instabilities [9, 12]. Instabilities caused by van der Waals interactions are sometimes called "spinodal" instabilities because the mathematical analysis is formally analogous to the analysis of the more familiar chemical spinodal decomposition [12]. Nucleated and spinodal dewetting may be simultaneously active as shown for liquid thins films of polymers [17], metals [13], and collagen solutions [18].

PCMs are generally embedded in a larger device or at least capped with a material that remains solid during the rapid heating used to induce the phase transformation, e.g. the ultrathin amorphous silicon nitride layer in Figure 1c. The presence of a solid capping layer on the PCM introduces another layer of complexity. Capping layers change the physical interactions, such as interfacial energies and van der Waals interactions, and introduce new factors, such as mechanical properties and stress state, in the solid capping material $[23,24]$. The presence of a solid cap may suppress some instabilities $[22,24]$ but, if subject to compressive stresses, may 
induce instabilities $[19,21,24]$. The need to better understand the complex physical mechanisms causing instabilities in nanostructured PCMs during switching motivates experimental observations of their development during laser heating in an effort to distinguish the different mechanisms of instability development.

We observe the development of morphological instabilities in situ, on the nanosecond scale, in capped GeTe films during laser heat treatment. GeTe is an important PCM, because its high crystallization temperature, $\mathrm{T}_{\mathrm{x}}$, relative to other commonly used PCMs $[25,26]$ and rapid switching speed [26-28] make it attractive for random access memory applications. GeTe has been the subject of numerous theoretical and experimental studies including several examining the crystallization behavior of amorphous thin films during isothermal [29-31] and laser [2, 3] heating and morphological changes have been seen during switching of GeTe nanowires [5]. Here we focus on GeTe, but we have observed similar instabilities in similarly prepared and treated $\mathrm{Ge}_{2} \mathrm{Sb}_{2} \mathrm{Te}_{5}$ and $\mathrm{GaSb}$ thin films. In situ observations allow us to differentiate dewetting mechanisms that would be difficult to distinguish in post mortem analysis. The microstructural changes that occur during laser heating would be impossible to observe with most in situ imaging techniques because they occur on the nanosecond to microsecond time scale and on a nanometer length scale.

\section{Experimental details}

We use the dynamic TEM (DTEM), a pulsed photoemission TEM at Lawrence Livermore National Laboratory, to image the GeTe during the development of morphological instabilities. The DTEM achieves nanosecond-scale time-resolved imaging of irreversible processes in condensed phases with intense photo-emitted electron pulses. During a time-resolved experiment, a reaction is initiated in the specimen with a "pump" laser pulse. A pulse from 
another laser, which is timed against the pump laser pulse in an operator-defined delay, strikes the cathode inducing a burst of $\sim 10^{10}$ photoemitted electrons, enough to form an image or diffraction pattern of a transient state of the material. The duration of the electron pulse passing through the reacting specimen defines the temporal resolution of the experiment. The DTEM has been used to study laser-induced crystallization in PCMs [3, 8, 32], rapidly-driven phase transformations in metals [33, 34], and semiconductors [35], and has been used to verify that spinodal dewetting is the controlling physical mechanism in the dewetting of ultrathin liquid Ni films [36]. The design and operation of the DTEM have been described elsewhere [37].

Morphological instabilities were induced by laser-heating as-deposited amorphous films of $30 \mathrm{~nm}$ GeTe on a Si support with a $20 \mathrm{~nm}$ thick amorphous silicon nitride window $(0.25 \times 0.25$ $\mathrm{mm}$ ) and $8 \mathrm{~nm}$ silica capping layer. GeTe was deposited at room temperature using magnetron sputtering. A target with a nominal composition of Ge:Te $=50: 50$ atomic $\%$ was used. Rutherford backscattering spectrometry revealed the film composition to be Ge $50.7 \pm 2.0$ atomic $\%$ and Te $49.3 \pm 2.0$ atomic $\%$. The specimen was heated with a neodymium-doped

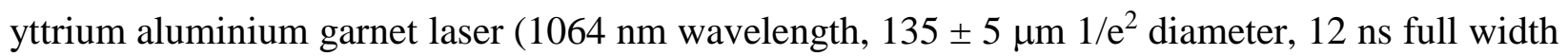
at half maximum pulse duration). The spot size on the specimen is larger than practical for memory devices, but the large, uniformly thick heated area is ideal for making observations that allow differentiation between different dewetting mechanisms. Laser heating was induced with 4.0 to $5.2 \mu \mathrm{J}$ pulses. The higher energies were used to allow the area of the specimen within the field of view to remain fluid for hundreds of nanoseconds, allowing thickness variations and dewetting to develop to a degree that they could be more easily observed. Bright field TEM images generated from 15-ns electron pulses were recorded before, during, and after laser heat treatment. The electron probe pulses and pump laser were aligned such that area where the 
specimen received the greatest laser intensity was imaged. Selected areas of laser-treated specimens were cut with a focused ion beam (FIB) to form thin cross-sections for post-mortem imaging in a conventional TEM.

Direct temperature measurements are not experimentally accessible for most laser heating experiments, but temperatures have been modeled [3]. Spatio-temporal temperature profiles caused by laser-specimen interactions and heat flow were developed using a commercially available finite element analysis modeling software (COMSOL Multiphysics) the details of which are available in the supplemental material of reference [3]. Simulations of temperatures at the center of the laser spot where the specimens were imaged are shown in Figure 2. The temperature rises rapidly during the laser pulse, but the cooling (governed by two-dimensional heat conduction in the suspended thin film) takes many microseconds. Heating due to the enthalpy of crystallization is not included in the model; it has been estimated that this could locally increase the temperature by $\sim 180 \mathrm{~K}$ near the crystallization front [3].

\section{Results and discussion}

Figure 3 shows morphological changes developing during crystallization of GeTe induced by a $4.0 \mu \mathrm{J}$ laser pulse. The development of the thickness variations occurs simultaneously with crystallization and the development of a hole, which was most likely nucleated at a pre-existing defect such as an impurity inclusion. The thickness variations run roughly parallel to the grain boundaries of four large GeTe grains at the top and center of Figure 3c and perpendicular to the circular rim of a dewet region in the lower right corner of the image. The morphology of the thickness variations was confirmed by TEM imaging of focused ion beam (FIB) cross sections (Figure 3d). The silicon nitride membrane forming the window of the Si TEM grid remained flat and the thickness variations in the GeTe that developed with distinctly regular, sinusoidal 
character were accommodated by displacement of the silica capping layer which remained conformal to the GeTe. In this case, in addition to the morphological changes, there are concomitant processes, e.g. phase transformations with accompanying changes in volume and crystal structure.

To allow observation of instabilities in the absence of crystallization, higher laser energies were used and $15 \mathrm{~ns}$ images were taken $500 \mathrm{~ns}$ after peak laser exposure of the specimen. Since the amorphous material is heated to higher temperatures, the nucleation rate of the crystalline phase at the center of the laser spot remains very low and the amorphous phase remains fluid for hundreds of nanoseconds. Figure 4 (a, c, e, g) show GeTe 500 ns after laser exposure for several laser energies. The same areas are shown minutes later in conventional TEM images (b, d, f, h). Instabilities of different types are seen to develop. The images in Figure 4 are $512 \times 512$ pixel selections capturing the area heated by the center of the laser spot.

Circular holes in the GeTe grow in Figure 4 (a, e); they are likely nucleated at inclusions in the films that are visible at the center of the holes in these time resolved images. Dewetting may have nucleated at these points either because of a local increase in temperature from increased laser absorption at the defects or due to a change to the energetic barrier to nucleation, i.e. alteration of the balance of the interfacial energies. The nucleated hole in Figure 4a has a larger radius in the image taken after crystallization (4b); the crystallization of the film presumably halted the further retraction of the edge of the hole. In addition to the nucleated hole, the $4.3 \mu \mathrm{J}$ laser shot caused regular thickness variations (Fig. 4b) that are not visible yet at 500 ns. The direction of the thickness variations appears to be biased by the nucleated hole as in the example shown in Figure 3c.

Figure 4 (c, g) show time resolved images of thin film instabilities in the absence of 
nucleated holes. Thickness variations in both cases are already apparent at $500 \mathrm{~ns}$ after laser heating. In the final, post mortem crystallized microstructures (Fig. $4(\mathrm{~d}, \mathrm{~h})$ ) both the amplitude and the wavelength of the thickness variations are significantly greater than at $500 \mathrm{~ns}$. For the 5.2 $\mu \mathrm{J}$ laser shot, the amplitude of the thickness variations increased to the point that the GeTe dewet in the troughs of the film leaving bare lines on the silicon nitride support. A fast Fourier transform (FFT) was taken of each image (Fig. 4 insets). The peak intensity of each rotationally averaged FFT in the pulsed images was taken as a measure of the characteristic wavelength, $\lambda_{\mathrm{m}}$, of the instability at $500 \mathrm{~ns}$ after laser heating. At $500 \mathrm{~ns}, \lambda_{\mathrm{m}}$ was found to be $320 \mathrm{~nm}$ and $290 \mathrm{~nm}$ for the $5.2 \mu \mathrm{J}$ and $4.6 \mu \mathrm{J}$ shots, respectively. The amplitude of the instabilities for the $4.2 \mu \mathrm{J}$ is too small at $500 \mathrm{~ns}$ to measure $\lambda_{\mathrm{m}}$. In the images of the final microstructure, the wavelength of the thickness variations has coarsened to $500 \mathrm{~nm}, 500 \mathrm{~nm}$, and $390 \mathrm{~nm}$ at the time of crystallization for the $5.2 \mu \mathrm{J}, 4.6 \mu \mathrm{J}$, and $4.2 \mu \mathrm{J}$ shots respectively. The coarsening of the wavelength may be driven by capillary effects unrelated to the mechanisms initiating the instability, so having measurements in the early stages of morphological development is important.

We have made estimates of $\lambda_{\mathrm{m}}$ produced either purely by van der Waals forces or by mechanical destabilization (see Appendix). The estimate for van der Waals driven instabilities is $\sim 140 \mu \mathrm{m}$ and for a mechanical buckling instability in the solid capping film it is $0.8 \mu \mathrm{m}$. Both estimates are larger than the wavelength of the instabilities observed here, but the estimate for the van der Waals induced instabilities is off by more than two orders of magnitude, while the estimate for mechanical instabilities is within a factor of three. This strongly suggests that mechanical instabilities are more important in the driving the non-nucleated thin films instabilities in this system, though it does not exclude an influence of van der Waals forces. From 
these initial experimental results, we are unable to quantify the relative influence of the van der Waals forces and mechanical (de)stabilization of the solid capping film on the $\lambda_{\mathrm{m}}$ in this system given the complexity of their interactions [24]. However, it has been shown for uncapped polystyrene that by varying the liquid film and substrate thickness, different mechanisms of thin film instabilities may be distinguished [20]. Since the van der Waals and mechanical forces scale differently with changing thickness of the fluid and capping layers, similar experiments to differentiate the relative importance of these mechanisms are possible. Since the characteristic amplitude, wavelength, and growth rate of linearized models of non-nucleated thin film instabilities apply to the earliest stages of film breakup [9, 12, 24], characterization and kinetic data from the early stages of development is necessary [17] and the nanosecond time-resolved in situ experiments described here will be instrumental in this characterization.

As a final observation, the behavior the GeTe film after a $5.0 \mu \mathrm{J}$ laser pulse is distinctly different from what occurs for higher $(5.2 \mu \mathrm{J})$ and lower $(4.6 \mu \mathrm{J})$ energy laser shots. The behavior may appear anomalous if only the final microstructure is observed, but the timeresolved images give great insight in explaining the apparently anomalous behavior. The nucleated dewetting initiates very soon after laser heating and by $500 \mathrm{~ns}$ some of the circular patches bare of GeTe are more than $2 \mu \mathrm{m}$ in diameter. It is the early development of the bare patches and the rapid retraction of the edges of the GeTe film that leads to a more advanced final state of dewetting. The final state consists of isolated drops of GeTe. Nucleated dewetting results in a more disrupted final state of the thin film compared to the results from purely non-nucleated dewetting at similar laser energies because of the earlier pinch-off of the GeTe film. It is useful to note however that non-nucleated dewetting may have a similar final microstructure, c.f. Figures $4(\mathrm{e}, \mathrm{f})$ and Figure $5(\mathrm{a}, \mathrm{b})$, thus the in situ experiments allow differentiation of dewetting 
mechanisms that may not be obvious from post mortem characterization.

\section{Conclusion}

We have imaged thin amorphous PCM in the time between laser heating and crystallization to capture early stages of morphological instabilities. The dewetting develops both from nucleation of bare patches and from spinodal instabilities. Although the spinodal instabilities may arise from either van der Waals interactions or a mechanical instabilities, the length scale of the observed instabilities during early stages of development indicate that mechanical instabilities are more important for driving the early stages of instabilities in these experiments. Further experiments are planned to quantify the relative importance of mechanical stresses and van der Waals forces in the destabilization of PCM thin films.

\section{Acknowledgements}

This work performed under the auspices of the U.S. Department of Energy, Office of Basic Energy Sciences, Division of Materials Sciences and Engineering by Lawrence Livermore National Laboratory under Contract DE-AC52-07NA27344. We thank P. Tchoulfian for performing ex situ laser experiments and E.A. Delenia for preparation of the FIB cross section.

\section{Appendix: Estimation of characteristic wavelength of instabilities}

The characteristic wavelength, $\lambda_{\mathrm{m}}$, or the wavelength that has the maximum growth rate of it's amplitude, is estimated here for two cases of instabilities: (1) instabilities arising purely from van der Waal's interactions and (2) instabilities arising purely from a mechanical instability due to compressive stresses developed in the solid capping layer during laser heating.

From Vrij and Overbeek [9], we estimate $\lambda_{\mathrm{m}}$ due to van der Waal's interactions:

$$
\lambda_{m, v d W}=\sqrt{\frac{8 \pi^{3} \gamma h^{4}}{A}}
$$


where $\gamma$ is the interfacial energy, $h$ is the initial thickness of the GeTe layer, and $A$ is the effective Hamaker constant. The Hamaker constant for two media interacting across a third condensed medium (here the support and capping materials interacting across the GeTe) will vary with the polarizabity of the materials but is expected to be on the order of $10^{-20}$ for most materials [38]. Using $h=30 \mathrm{~nm}$ for the GeTe thickness and representative values of $\gamma=1 \mathrm{~J} / \mathrm{m}^{2}$ and $A=10^{-20} \mathrm{~J}$, yields $\lambda_{m, \mathrm{vdW}}=140 \mu \mathrm{m}$, orders of magnitude off from the $\sim 0.30 \mu \mathrm{m}$ observed.

For the mechanical stresses, we assume that the silicon nitride membrane may be treated as a rigid support; the supports used are under tensile stress and remain relatively flat even during the development of significant thickness variations in the GeTe as seen in the cross section images, c.f. Figure 1d (main text). We assume that the amorphous capping layer initial is stress free and that in the regions where the GeTe has been heated adequately to flow like a liquid, the capping layer buckles to accommodate compressive stresses that develop due to laser heating.

From Sridhar et al. [21], we estimate the characteristic buckling wavelength of a solid thin film on a viscous layer due to compressive stress. For limit of a thin viscous layer the characteristic wavelength is:

$$
\lambda_{m, \text { mech }}=\frac{2 \pi h_{c a p}}{\sqrt{\frac{2}{3} 12 \varepsilon_{o}(1+v)}}
$$

where $h_{\text {cap }}$ is the thickness of the solid capping layer, $\varepsilon_{0}$ is the strain in the solid thin film, and $v$ is the Poisson ratio of the solid film. We use the properties of fused silica to make our estimates: $v=0.17$ and a coefficient of thermal expansion $5.5 \times 10^{-7} /{ }^{\circ} \mathrm{C}$ to estimate the strain for a change in temperature of $500^{\circ} \mathrm{C}$. For a capping layer thickness of $8 \mathrm{~nm}$ we find $\lambda_{m \text {,mech }}$ to be $0.8 \mu \mathrm{m}$, larger than the observed wavelength of the thickness variations, but within a factor of three.

Both the van der Waals and mechanical instability estimates are larger than the wavelength 
of the instabilities observed here. Since both estimates are simplified views of a complex process that could include both mechanisms, and since only representative values were taken for some physical parameters in the models, it is not surprising that neither estimate for matches the experimental observations perfectly. However, the estimate for the van der Waals induced instabilities is off by more than two orders of magnitude, while the estimate for mechanical instabilities is within a factor of three. This very strongly suggests that mechanical instabilities are more important in initially driving the non-nucleated thin film instabilities in this system, though it does not exclude an influence of van der Waals forces.

\section{References}

[1] S. Raoux, M. Wuttig, Phase Change Materials: Science and Applications, Springer Verlag, Berlin, 2009.

[2] E. Huber, E.E. Marinero, Laser-induced crystallization of amorphous GeTe - a time resolved study, Phys. Rev. B. 36 (1987) 1595-604.

[3] M.K. Santala, B.W. Reed, S. Raoux, T. Topuria, T. LaGrange, G.H. Campbell, Irreversible reactions studied with nanosecond TEM movies: laser crystallization of phase change materials, Appl. Phys. Lett. 102 (2013) 174105.

[4] V. Weidenhof, I. Friedrich, S. Ziegler, M. Wuttig, Atomic force microscopy study of laser induced phase transitions in $\mathrm{Ge}_{2} \mathrm{Sb}_{2} \mathrm{Te}_{5}$, J. Appl. Phys. 86 (1999) 5879-87.

[5] S. Meister, H. Peng, K. McIlwrath, K. Jarausch, X.F. Zhang, Y. Cui, Synthesis and characterization of phase-change nanowires, Nano Lett. 6 (2006) 1514-7.

[6] B.J. Kooi, J.L.M. Oosthoek, M.A. Verheijen, M. Kaiser, F.J. Jedema, D.J. Gravesteijn, Nanostructure-property relations for phase-change random access memory (PCRAM) line cells, Phys. Status Solidi B. 249 (2012) 1972-7.

[7] P. Kivits, R. Debont, B. Jacobs, P. Zalm, The hole formation process in tellurium layers for optical-data storage, Thin Solid Films. 87 (1982) 215-31.

[8] M.K. Santala, B.W. Reed, S. Raoux, T. Topuria, T. LaGrange, G.H. Campbell, Nanosecondscale time-resolved electron imaging during laser crystallization of GeTe, Phys. Status Solidi B. 249 (2012) 1907-13.

[9] A. Vrij, J.T. Overbeek, Rupture of thin films due to spontaneous fluctuations in thickness, J. Am. Chem. Soc. 90 (1968) 3074-8.

[10] F. Brochard Wyart, J. Daillant, Drying of solids wetted by thin liquid films, Can. J. Phys. 68 (1990) 1084-8.

[11] H.S. Kheshgi, L.E. Scriven, Dewetting - nucleation and growth of dry regions, Chem. Eng. Sci. 46 (1991) 519-26.

[12] V.S. Mitlin, Dewtting of solid-surface - analogy with spinodal decomposition, J. Colloid Interface Sci. 156 (1993) 491-7.

[13] J. Bischof, D. Scherer, S. Herminghaus, P. Leiderer, Dewetting modes of thin metallic films: Nucleation of holes and spinodal dewetting, Phys. Rev. Lett. 77 (1996) 1536-9. 
[14] A. Oron, S.H. Davis, S.G. Bankoff, Long-scale evolution of thin liquid films, Rev. Mod. Phys. 69 (1997) 931-80.

[15] S. Herminghaus, K. Jacobs, K. Mecke, J. Bischof, A. Fery, M. Ibn-Elhaj, S. Schlagowski, Spinodal dewetting in liquid crystal and liquid metal films, Science. 282 (1998) 916-9.

[16] A. Sharma, R. Khanna, Pattern formation in unstable thin liquid films, Phys. Rev. Lett. 81 (1998) 3463-6.

[17] R. Xie, A. Karim, J.F. Douglas, C.C. Han, R.A. Weiss, Spinodal dewetting of thin polymer films, Phys. Rev. Lett. 81 (1998) 1251-4.

[18] U. Thiele, M. Mertig, W. Pompe, Dewetting of an evaporating thin liquid film: Heterogeneous nucleation and surface instability, Phys. Rev. Lett. 80 (1998) 2869-72.

[19] N. Bowden, S. Brittain, A.G. Evans, J.W. Hutchinson, G.M. Whitesides, Spontaneous formation of ordered structures in thin films of metals supported on an elastomeric polymer, Nature. 303 (1998) 146-9.

[20] R. Seemann, S. Herminghaus, K. Jacobs, Dewetting patterns and molecular forces: A reconciliation, Phys. Rev. Lett. 86 (2001) 5534-7.

[21] N. Sridhar, D.J. Srolovitz, Z. Suo, Kinetics of buckling of a compressed film on a viscous substrate, Appl. Phys. Lett. 78 (2001) 2482-4.

[22] R. Huang, Z. Suo, Wrinkling of a compressed elastic film on a viscous layer, J. Appl. Phys. 91 (2002) 1135-42.

[23] P.J. Yoo, H.H. Lee, Evolution of a stress-driven pattern in thin bilayer films: Spinodal wrinkling, Phys. Rev. Lett. 91 (2003) 154502.

[24] R. Huang, Z. Suo, Very thin solid-on-liquid structures: the interplay of flexural rigidity, membrane force, and interfacial force, Thin Solid Films. 429 (2003) 273-81.

[25] S. Raoux, B. Munoz, H.-Y. Cheng, J.L. Jordan-Sweet, Phase transitions in Ge-Te phase change materials studied by time-resolved x-ray diffraction, Appl. Phys. Lett. 95 (2009) 143118.

[26] G. Bruns, P. Merkelbach, C. Schlockermann, M. Salinga, M. Wuttig, T.D. Happ, J.B. Philipp, M. Kund, Nanosecond switching in GeTe phase change memory cells, Appl. Phys. Lett. 95 (2009) 043108.

[27] M. Chen, K.A. Rubin, R.W. Barton, Compound materials for reversible, phase-change optical-data storage, Appl. Phys. Lett. 49 (1986) 502-4.

[28] S. Raoux, H.Y. Cheng, M.A. Caldwell, H.S.P. Wong, Crystallization times of Ge-Te phase change materials as a function of composition, Appl. Phys. Lett. 95 (2009) 071910.

[29] Q.M. Lu, M. Libera, Microstructural measurements of amorphous GeTe crystallization by hot-stage optical microscopy, J. Appl. Phys. 77 (1995) 517-21.

[30] A.M. Mio, E. Carria, G. D'Arrigo, S. Gibilisco, M. Miritello, M.G. Grimaldi, E. Rimini, Nucleation and grain growth in as deposited and ion implanted GeTe thin films, J. Non-Crystal. Solids. 357 (2011) 2197-201.

[31] A. Bastard, J.C. Bastien, B. Hyot, S. Lhostis, F. Mompiou, C. Bonafos, G. Servanton, C. Borowiak, F. Lorut, N. Bicais-Lepinay, A. Toffoli, C. Sandhya, A. Fantini, L. Perniola, E. Gourvest, S. Maitrejean, A. Roule, V. Sousa, D. Bensahel, B. Andre, Crystallization study of "melt quenched" amorphous GeTe by transmission electron microscopy for phase change memory applications, Appl. Phys. Lett. 99 (2011) 243103.

[32] M.K. Santala, B.W. Reed, T. Topuria, S. Raoux, S. Meister, Y. Cui, T. LaGrange, G.H. Campbell, N.D. Browning, Nanosecond in situ transmission electron microscope studies of the reversible $\mathrm{Ge}_{2} \mathrm{Sb}_{2} \mathrm{Te}_{5}$ crystalline $\Leftrightarrow$ amorphous phase transformation, J. Appl. Phys. 111 (2012) 024309. 
[33] J.S. Kim, T. LaGrange, B.W. Reed, R. Knepper, T.P. Weihs, N.D. Browning, G.H. Campbell, Direct characterization of phase transformations and morphologies in moving reaction zones in $\mathrm{Al} / \mathrm{Ni}$ nanolaminates using dynamic transmission electron microscopy, Acta Mater. 59 (2011) 3571-80.

[34] J. McKeown, A. Kulovits, C. Liu, K. Zweiacker, B.W. Reed, T. LaGrange, J.M.K. Wiezorek, G.H. Campbell, In situ transmission electron microscopy of crystal growth-mode tranisitions during rapid solidifcation of a hypoeutectic Al-Cu alloy, Acta Mater. 65 (2014) 5668 .

[35] L. Nikolova, T. LaGrange, M.J. Stern, J.M. MacLeod, B.W. Reed, H. Ibrahim, G.H. Campbell, F. Rosei, B.J. Siwick, Complex crystallization dynamics in amorphous germanium observed with dynamic transmission electron microscopy, Phys. Rev. B. 87 (2013) 064105. [36] J.T. McKeown, N.A. Roberts, J.D. Fowlkes, Y. Wu, T. LaGrange, B.W. Reed, G.H. Campbell, P.D. Rack, Real-time observation of nanosecond liquid-phase assembly of nickel nanoparticles via pulsed-laser heating, Langmuir. 28 (2012) 17168-75.

[37] M.R. Armstrong, K. Boyden, N.D. Browning, G.H. Campbell, J.D. Colvin, W.J. DeHope, A.M. Frank, D.J. Gibson, F. Hartemann, J.S. Kim, W.E. King, T.B. LaGrange, B.J. Pyke, B.W. Reed, R.M. Shuttlesworth, B.C. Stuart, B.R. Torralva, Practical considerations for high spatial and temporal resolution dynamic transmission electron microscopy, Ultramicrosc. 107 (2007) $356-67$.

[38] J. Isrealachvili, Intermolecular and Surface Forces, Elsevier Ltd., London, 1991. 


\section{Figure Captions}

Figure 1. Conventional ex situ TEM images of (a) laser-crystallized marks in an amorphous $\mathrm{Ge}_{2} \mathrm{Sb}_{2} \mathrm{Te}_{5} \mathrm{thin}_{\text {film }}$ and (b) a laser-amorphized mark in a crystalline film. Within the amorphous mark, darker areas are thinner and lighter areas thicker. High-angle annular dark field scanning TEM of the area in 1b (not shown) and (c) TEM of a FIB cross section of a laser-amorphized area, similar to that shown in (b), confirm the contrast changes in the amorphous region are due to thickness variations. Similar thickness variations occur within the crystalline marks and holes develop in the $\mathrm{Ge}_{2} \mathrm{Sb}_{2} \mathrm{Te}_{5}$ with increased laser energy and pulse duration ((a), bottom).

Figure 2 The simulated temperature during $1064 \mathrm{~nm}$ wavelength laser heating of silica-capped GeTe on a $250 \mu \mathrm{m}$ silicon nitride TEM window. The temperatures plotted are for the point at the center of the Gaussian laser pulse and are given for the range of energies used in the experiments. Heating is rapid during the first few nanoseconds while the laser is on. After the laser pulse the local temperature remains steady for microseconds.

Figure 3 TEM images show morphological instabilities and dewetting in a laser crystallized GeTe thin film. The asdeposited $30 \mathrm{~nm}$ GeTe film (a) appears featureless. A $15 \mathrm{~ns}$ pulsed electron image $1400 \mathrm{~ns}$ after the peak in laser heating (b) shows crystalline grains (lighter ovals with very dark bend contours) growing into the amorphous film. The arrow marks dark circular edge of an area that has partially dewet. The same region is shown minutes later in a conventional image (c), the evenly spaced light and medium grey striations radiating from the circular flaw in the lower right and running parallel to the grain boundaries in the large grains in the center of the image are due to thickness variations. A TEM image of a focused ion beam cross section (d) of the film confirms the striations are due to thickness variations and shows their regular sinusoidal morphology.

Figure 4 Time-resolved (15 ns) images of GeTe films 500 ns after laser exposure for four different laser energies (top row). The same areas were imaged minutes after laser exposure using conventional bright field imaging (bottom row). All images are to the same scale. Images are cropped to 512 x 512 pixels showing the center of the laser affected area. Insets are FFT-generated power spectra, identically cropped to highlight the central features.

Figure 5 Dewetting $500 \mathrm{~ns}$ after a $532 \mathrm{~nm}$ laser pulse (a) and the same are minutes later (b). The dewetting appears to occur through a non-nucleated spinodal process but the final microstructure is similar to figure $4 \mathrm{f}$, where nucleated dewetting appeared to control morphological development. In situ characterization distinguishes mechanisms not apparent in post mortem characterization. The laser energy is lower than in figure 4 but GeTe absorbs more in the green than in the ultraviolet part of the spectrum. Images and power spectra are cropped as in figure 4 . 


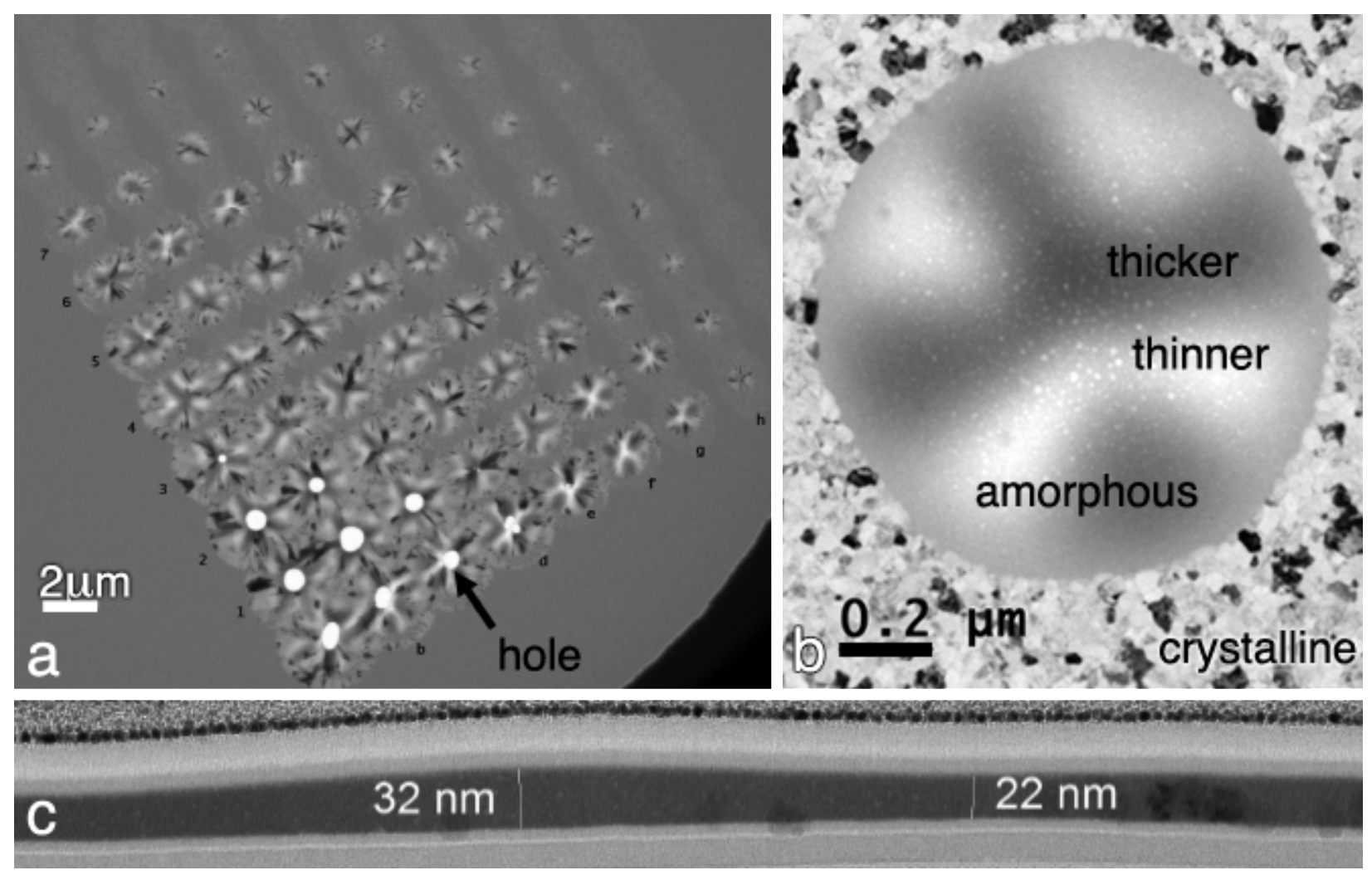




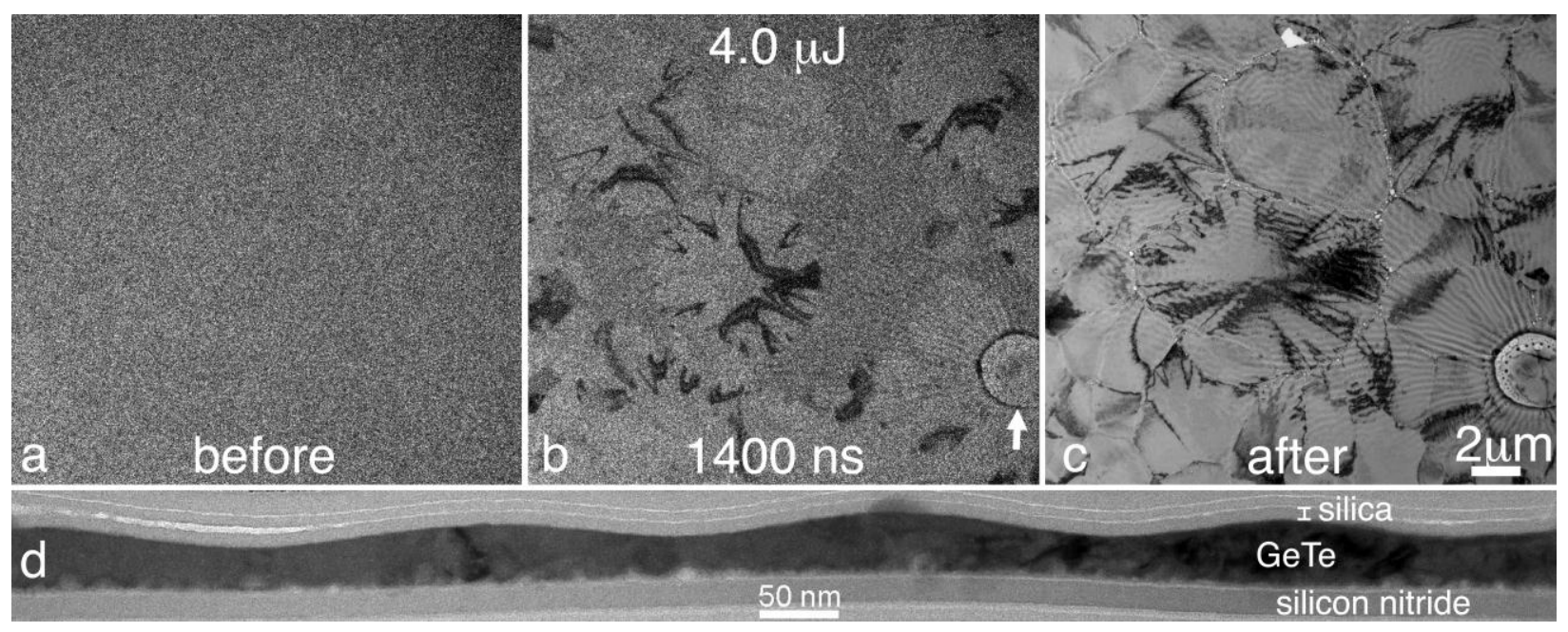




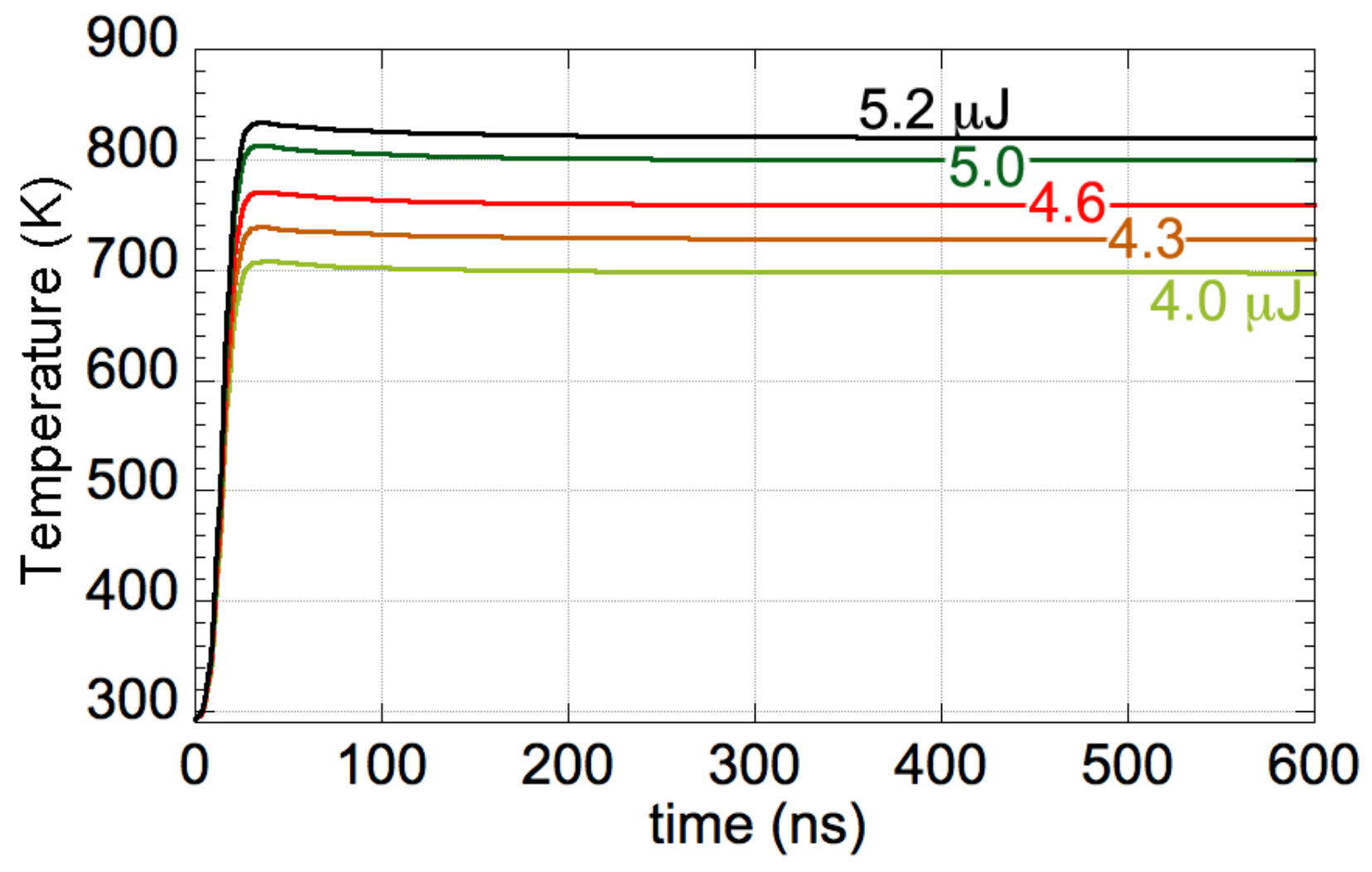




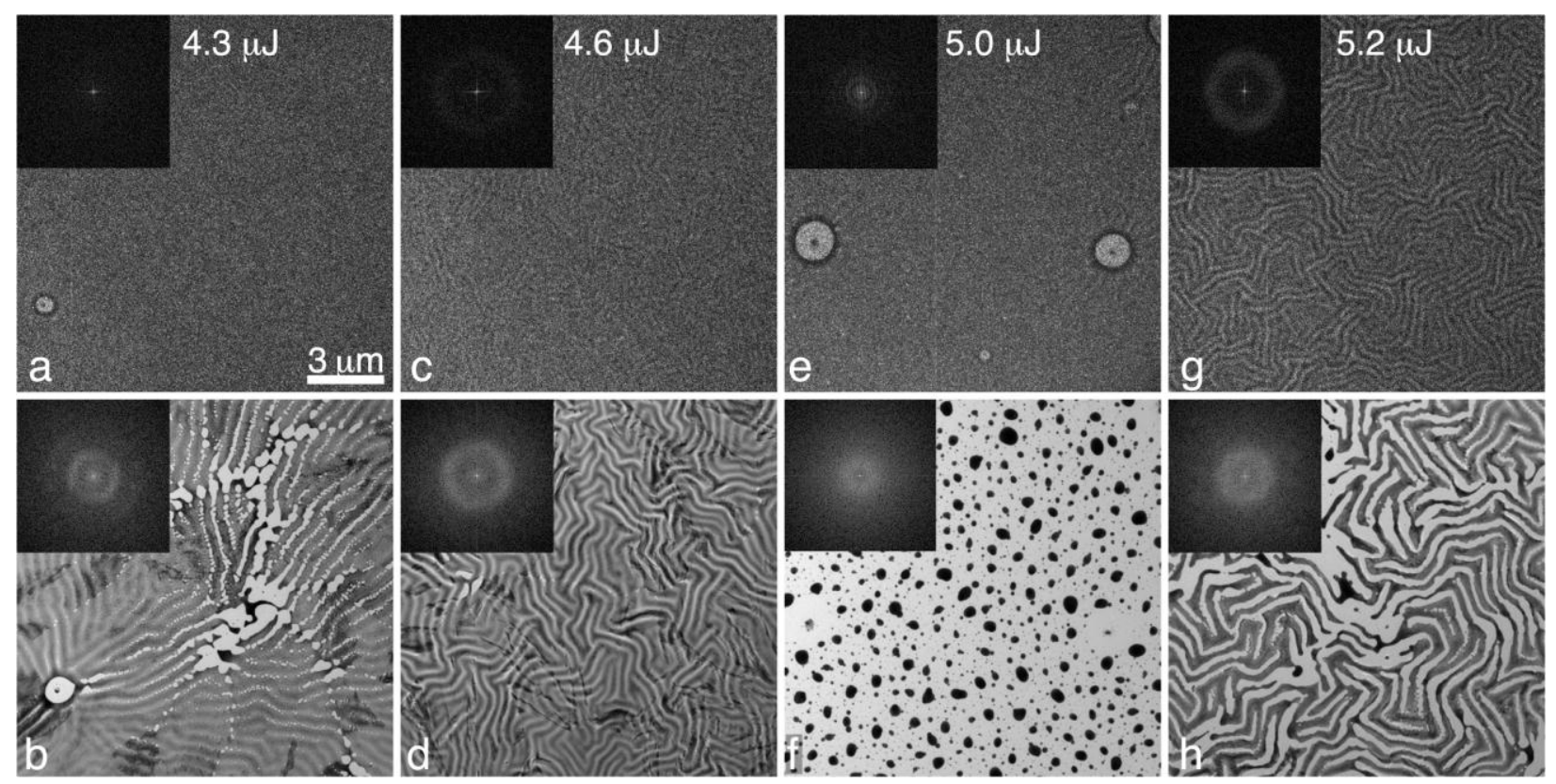




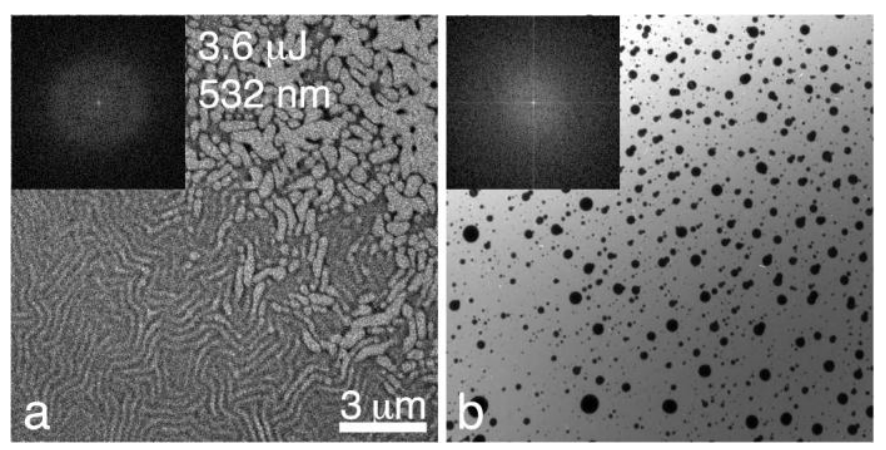

